\title{
Soluble CD59-Antigen Levels are Increased in Renal Disease Plasma
}

\author{
Oluseyi Adewale Vanderpuye \\ Department of Criminal Justice and Forensic Science, Albany State University, Albany, Georgia, USA
}

Received 2013-07-04, Revised 2013-07-25; Accepted 2013-08-10

\begin{abstract}
Cluster of Differentiation antigen 59 (CD59) is a glycosyl phosphatidylinositol-linked protein that protects cells from damage by lytic terminal complexes of complement. Soluble forms of CD59 in serum have been little investigated in disease states. In the present study, by using a sandwich ELISA, reactivities with antibodies to CD59 were compared for sera and plasma from normal individuals and those with renal, leukemia, heart, liver and lung diseases. In all of 44 renal patients, reactivities were up to three-fold higher than those of normals and patients with other conditions. The soluble CD59-related antigen displayed epitopes recognized by an anti-CD59 rabbit antiserum and CD59 monoclonal antibodies MEM-43 and YTH 53.1. Failure to bind the hydrophobic matrix phenyl Sepharose suggested that the CD59-related antigen lacked a membrane-anchoring structure. Although the cellular origin of soluble CD59-related antigen is unknown, some effect common to different renal diseases can promote increased plasma levels of this antigen. In two individuals, monitored before and after renal transplantation, plasma levels of CD59-related antigen decreased towards normal after receipt of a kidney.
\end{abstract}

Keywords: Complement, CD59, ELISA, Plasma, Renal Transplantation, Glycosyl Phosphatidylinositol

\section{INTRODUCTION}

CD59 is a glycoprotein of $18-21 \mathrm{kDa}$ that is expressed on cell surfaces of a wide variety of human cells. It regulates complement by binding to homologous C5b-8 proteins complex to prevent binding of Complement component 9 and assembly of the cell lysing forms of the membrane attack complex of complement (Zhu et al., 2008). CD59 has been directly shown to protect autologous cells from attack by complement (Yang et al., 2011). Supporting evidence for a protective role for CD59 in general arises from reports of cellular damage occurring in cases of genetic CD59 deficiency, the promotion of susceptibility to complement by inhibitory antibodies to CD59 and the transfer of resistance to human complement by transfection of the CD59 gene into nonhuman cells (Yang et al., 2011; Tembhare et al., 2010). One study reported that the presence of CD59 provides protection from complement cooperative with that of CD55 which regulates complement at the level of C3 (Xu et al., 2006).
The scientific literature provides extensive evidence for the significant involvement of CD59 and the complement system in many diseases and conditions (Zipfel and Skerka, 2009). As one example, a decrease in CD59 expression occurred in human renal tubular epithelial and glomerular endothelial cells after exposure to Shiga toxin (Nishi et al., 2013). In myocardial infarction, CD59 is lost from myocytes in affected tissue concomitantly with C5b-9 deposition and serum levels of CD59 are increased in the acute phase (Vakeva et al., 1992; 2000).

In relation to renal disease in particular, there is also considerable documentation of the significance of complement and complement regulators. Recent reviews described the importance of dysregulation of complement in different kidney diseases (Berger and Daha, 2007; Heeringa and Cohen, 2012). Immunohistological studies showed an increase in glomerular CD59 in cases of lupus nephritis (Tamai et al., 1991). Another study found changes in the C5b-9 complex which is regulated by CD59 as well as in cell membrane 
CD59 in transplanted kidneys (Nishi et al., 2004). One case study of an individual with paraoxysmal nocturnal hemoglobinuria, in which cells are deficient in CD59 and CD55, reported associated kidney damage (Qi et al., 2011). Experimental studies in animal models of renal disease have also directly demonstrated roles for injury produced by activation of the complement system (He et al., 2005; Bao et al., 2009). In mice in which the genes for CD59 and another complement regulator CD55 have been knocked out, kidneys are more susceptible to renal ischemia-reperfusion injury (Miwa et al., 2013).

In view of the previously reported disease-linked changes in CD59 expression in the various circumstances mentioned above, it was hypothesized that plasma levels of CD59 are altered in kidney disease. There have been no previous studies on changes in serum or plasma CD59 in renal disease. The aim of the present study was to assess levels of CD59 in plasmas from individuals with renal disease and other conditions by using a sandwich ELISA developed in a previous study (Vanderpuye et al., 1993). This study found that increases in plasma CD59 occurred in individuals with end stage renal disease and that in two cases the elevated levels of plasma C59 decreased after kidney transplantation.

\section{MATERIALS AND METHODS}

Sandwich ELISAs of CD59 in serum or plasma were done as previously described (Vanderpuye et al., 1993). In brief, Immulon 2 microtiter plate wells (Dynatech, Alexandria, VA, USA) were coated with $50 \mu \mathrm{L}$ of rabbit anti-CD59 IgG (serum was gift of Dr C.J. Parker) at 5?g protein $\mathrm{ml}^{-1}$ in $0.2 \mathrm{M} \mathrm{Na}_{2} \mathrm{CO}_{3}, \mathrm{pH} 9.6$ for $16 \mathrm{~h}$ at $4{ }^{\circ} \mathrm{C}$. After blocking excess binding sites with $20 \mathrm{mg} \mathrm{mL}^{-1}$ BSA in $150 \mathrm{mM} \mathrm{NaCl}, 10 \mathrm{mM}$ sodium phosphate, $\mathrm{pH} 7.4$ (PBS), $50 \mu \mathrm{L}$ of plasma, serum or derived fractions were added and incubated for $1 \mathrm{~h}$ at $2528^{\circ} \mathrm{C}$. Wells were washed five times with PBS containing $0.1 \%(\mathrm{v} / \mathrm{v})$ Tween-20. Incubation with the detection antibody, monoclonal antibody MEM-43 to CD59 (Biodesign, Kennebunkport, MA, USA) $\left(0.1 \mu \mathrm{g} \mathrm{mL}^{-1}\right)$ was for $1 \mathrm{~h}$. The second antibody was peroxidase-conjugated antimouse $\mathrm{IgG}$ at $1 \mu \mathrm{g} \mathrm{g} \mathrm{mL} \mathrm{mL}^{-1}$ (Dako, Santa Barbara, CA, USA) and the tetramethyl benzidine substrate solution was from Kirkegaard and Perry Laboratories (Gaithersburg, MD, USA). In order to confirm that the ELISA was specific, negative controls were done for both the rabbit capture antibody and the monoclonal antibody used to detect the captured CD59. The polyclonal rabbit anti CD59 capture antibody was substituted with rabbit antibody to the serum protein orosomucoid (Dako). In addition, the monoclonal antibody to CD59 was substituted with isotype-matched monoclonal antibody (IgG2a) UPC-10 (from Sigma), of no known specificity. Use in the sandwich ELISA of either negative control, rabbit anti-orosomucoid or mouse monoclonal UPC-10 resulted in negligible absorbance values similar to those of blank wells lacking antibodies altogether.

Competition ELISAs for binding of anti-CD59, that involved erythrocytes as targets, were done as another method of assaying CD59 in plasma. The principle is that in the presence of a liquid containing CD59, monoclonal antibodies to CD59 will be prevented from binding from binding to immobilized erythrocytes to a degree dependent on the amount of CD59 present in the media in which binding interactions take place. The competition ELISAs for CD59 in plasma involved coating microtiter plate wells using $1 \mathrm{mg}$ protein $\mathrm{mL}^{-1}$ of erythrocyte membranes prepared by hypotonic lysis and centrifugation. Serial two-fold dilutions of a monoclonal antibodies MEM-43 or YTH53.1 that recognize CD59 were made from an initial concentration of $0.1 \mu \mathrm{g} \mathrm{mL}^{-1}$ in one of the following media: non-diluted human plasma, guinea pig plasma or $0.1 \mathrm{mg} \mathrm{mL}^{-1}$ erythrocyte membranes or PBS. The PBS and guinea pig plasma was a negative control. After incubation for $1 \mathrm{~h}$ with one of these primary antibodies, the wells were washed five times with PBSTween 20, incubated with peroxidase-conjugated antimouse IgG and developed as described above.

Renal ACD plasmas and sera were from 44 patients with end-stage renal disease who were awaiting kidney transplants at Methodist Hospital of Indiana. Other plasmas and sera were from candidates for 5 heart, 3 liver and 1 lung transplants and from 3 individuals with leukemia and 1 with lymphoma who were awaiting bone marrow transplantation. Normal plasmas and sera were from 25 healthy volunteers in laboratories at Methodist Hospital of Indiana.

\section{RESULTS}

\subsection{Optimization of Sandwich ELISA for CD59 in Plasma and Serum}

Sandwich ELISAs were previously used for measuring CD59 in various membranes after solubilization with the zwitterionic detergent CHAPS (Vanderpuye et al., 1993). Experiments were done to assess the specificity and sensitivity of the CD59 sandwich ELISA for plasma. Rabbit anti-CD59 IgG and as a negative control, rabbit anti-orosomucoid were used 
in varying concentrations to coat microtiter plates as capture antibodies for pooled human plasma. Monoclonal antibody MEM-43 was used to detect binding of CD59-related antigen and the results are shown in Fig. 1A. The optimal coating concentration range for rabbit anti-CD59 $\mathrm{IgG}$ capture of antigen was 5-19 ng $\mathrm{mL}^{-1}$. Negligible reactivity with MEM-43 occurred when rabbit anti-orosomucoid was the capture antibody. The monoclonal antibody UPC-10 when used as negative control for the MEM-43 antibody to CD59, did not display significant specific binding when either anti-CD59 or anti-orosomucoid was the capture antibody. Additionally, in another experiment, different dilutions of plasma and serum from the same individual were compared for reactivity in the CD59 ELISA and no significant difference was observed between serum and plasma (Fig. 1B).

\subsection{Titration of anti-CD59 Reactivity of Pooled Plasmas from Normals and Renal Patients}

Reactivities of different dilutions of plasmas pooled from 8 normal individuals and 8 with renal disease were compared in the CD59 sandwich ELISA to identify a dilution range suitable for comparison of plasmas and sera from these two groups (Fig. 2). Pooled plasmas from renal patients were found to contain significantly more CD59-related antigen than pooled plasmas from normals. At an eight-fold dilution, absorbances were 0.65 for normal plasma and 1.4 for renal plasma. At a dilution of 64-fold, normal plasma did not react positively while renal plasma produced an absorbance of approximately 0.75 . In subsequent comparisons, plasmas were diluted in the range 10-80 fold.

\subsection{Competition of Binding of anti-CD59 Antibodies YTH53.1 and MEM-43 by Plasmas From Normals and Renal Patients}

To confirm that soluble forms of CD59 were present in plasma, the ability of plasma to compete for the binding to human erythrocyte membranes of MEM-43 and YTH53.1, two monoclonal antibodies to CD59, was assessed. For the competition ELISA, antibodies to CD59 were serially diluted in two-fold serial increments into solutions containing a fixed amount of erythrocyte membranes, renal patient plasma, guinea pig plasma or PBS. Dilution into solutions containing human erythrocyte membranes provided a positive control of a medium containing CD59 while guinea pig plasma served as a negative control. Dilution into PBS provided a profile of the decrease in reactivity due to lowering antibody concentrations in the absence of a competitor. The results are shown in Fig. 3.

The binding of mouse monoclonal antibody MEM-43 to erythrocytes was essentially the same when diluted into PBS or guinea pig plasma, at 8 and 16-fold dilutions, reactivities were 50 and $25-35 \%$ those of undiluted antibody respectively. In contrast, reactivities of MEM-43 diluted 8 and 16-fold in solutions containing erythrocyte membranes or renal patient plasma were reduced to less than $10 \%$ that of controls (Fig. 3A). A similar experiment was done using the rat monoclonal antibody YTH53.1 (Fig. 3B). At a 16- fold dilution in PBS or guinea pig plasma, YTH53.1 reactivity with human erythrocytes was the same or slightly higher than for non-diluted antibody. For 8 and 16-fold dilutions in solutions containing human erythrocytes or renal patient plasma, reactivity of YTH53.1 was competed to less than $10 \%$ that of non-diluted antibody. The results suggested that renal patient plasma contains an antigen that binds two different CD59 monoclonal antibodies to prevent their reaction with erythrocyte CD59.

\subsection{Soluble CD59 in Patients with end Stage Renal Disease and Other Conditions}

Plasmas from normal individuals were compared in the CD59 sandwich ELISA with plasmas from individuals with various pathologic conditions to identify states in which soluble CD59-related antigen were altered. Sandwich ELISA using rabbit anti-CD59 capture antibodies and anti-CD59 MEM-43 monoclonal antibody was performed to determine CD59 levels (+/- standard error of mean) in two different pools of normal human plasma and plasmas from different conditions.

Comparisons are shown for plasmas from normals and individuals with end-stage renal disease (Fig. 4). The average absorbance value for 44 renal patients was 1.406 + - 0.207 (range 0.954-1.756) and the average absorbance for 25 normal plasmas was $0.492+/-0.115$ (range $0.285-0.684)$. These values were significantly different ( $\mathrm{t}$ test, $\mathrm{p}<0.0001$ ).

Soluble CD59-related antigen was also assessed in plasmas from patients awaiting heart, liver and lung transplantation (Table 1). Reactivities of plasmas from liver transplant candidates were in three cases 90 to $122 \%$ those of averaged normal plasmas and in one case were one third of normal. Plasma from the lung transplant candidate had $86 \%$ of the reactivity of pooled normal plasma. Renal patient plasma reactivities with anti-CD59 were two-fold or more higher than those of liver, lung and heart transplant candidates. 


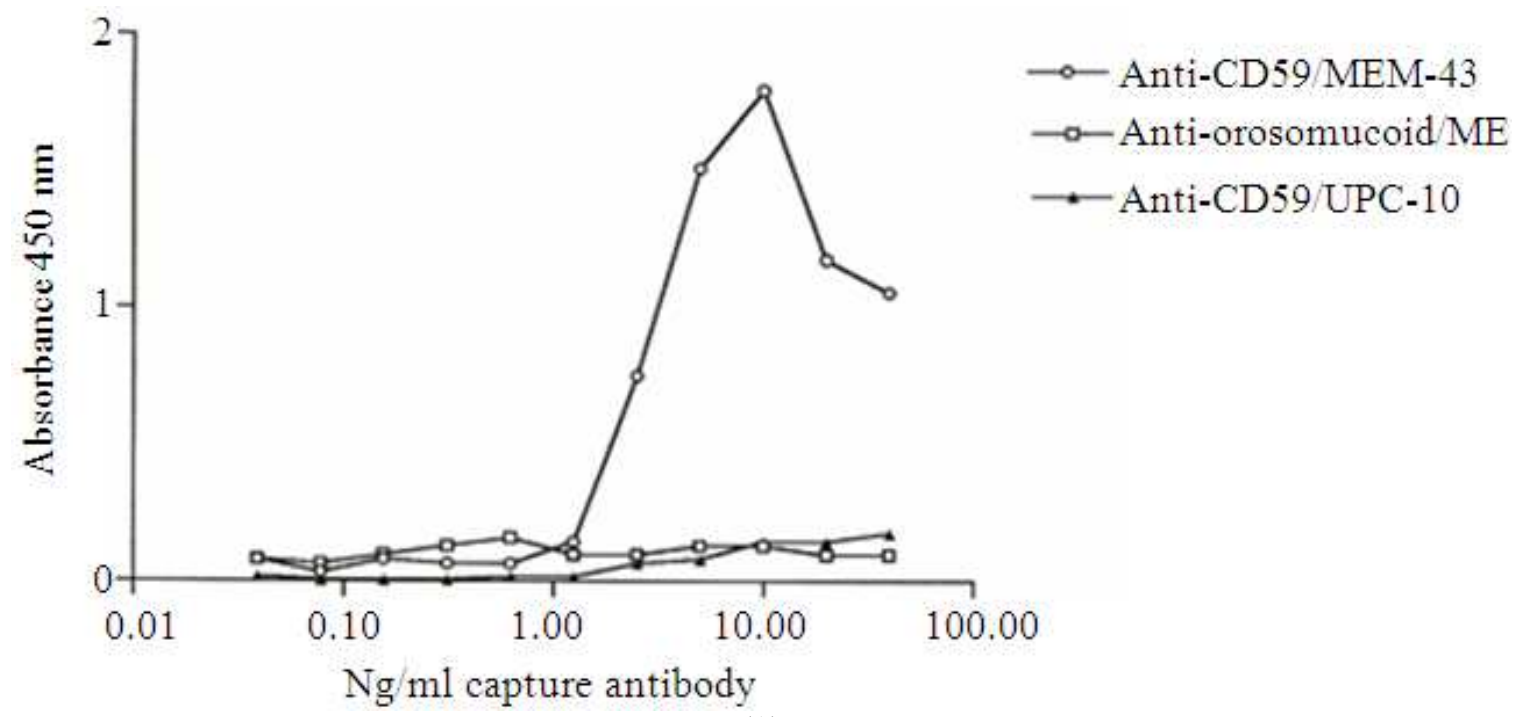

(A)

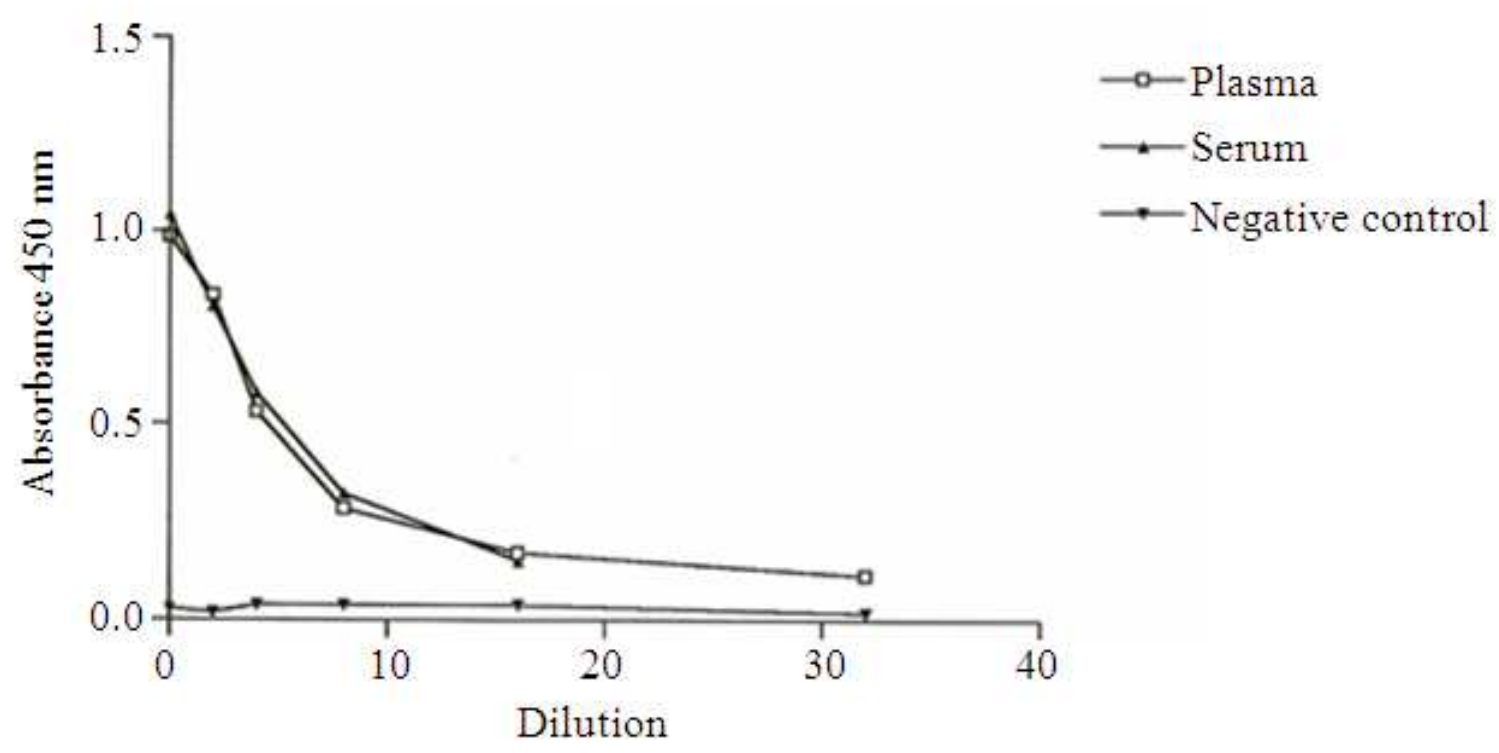

(B)

Fig. 1. (A) Optimization and specificity of sandwich ELISA for plasma CD59. Microtiterplate wells were coated with capture antibody rabbit anti-CD59 IgG or negative control antibody rabbit anti-orosomucoid, at varying concentrations and then incubated with human plasma. Monoclonal antibodies were added and followed by peroxidase-conjugated anti-mouse IgG and peroxidase substrate. Binding curves are shown for MEM-43 monoclonal antibody CD59 for proteins captured by rabbit anti-CD59 (open circles) or by rabbit anti-orosomucoid (open squares). Binding of isotype matched negative control monoclonal antibody UPC-10 (solid triangles) is shown for proteins captured by rabbit anti-CD59. (B). CD59related antigen is present in the same amount in plasma and serum from the same donor. Different concentrations (dilutions) of plasma (open squares) and serum (solid triangles) were assayed by sandwich ELISA by using rabbit antiCD59 and MEM-43 monoclonal antibody to CD59. Binding of isotype matched negative control monoclonal antibody UPC-10 is shown (inverted triangles) for plasma proteins captured by rabbit anti-CD59 


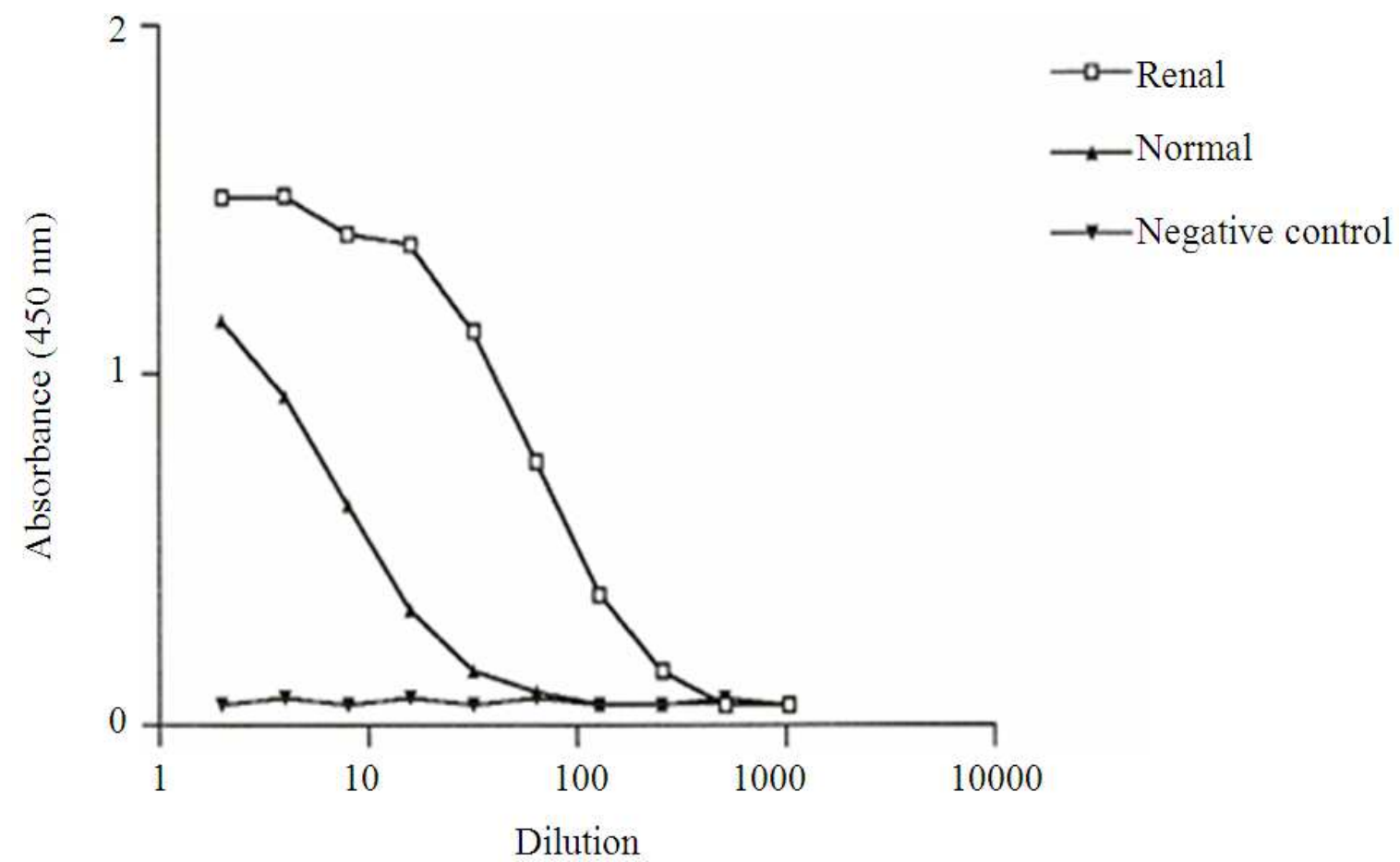

Fig. 2. Plasma from renal patients contains more CD59 than normal plasmas across different dilutions. Two fold serial dilutions were made of pooled plasma from renal patients (squares, curve 1) or pooled normal plasmas (triangle, curve 2). The diluted plasma samples were assayed by CD59 sandwich ELISA by using rabbit anti-CD59 as capture antibody and MEM-43 monoclonal antibody for detection along with peroxidase-conjugated anti-mouse IgG and peroxidase colorimetric substrate. Binding of negative control monoclonal antibody UPC-10 in combination with rabbit anti CD59 is shown in inverted triangles (curve 3)

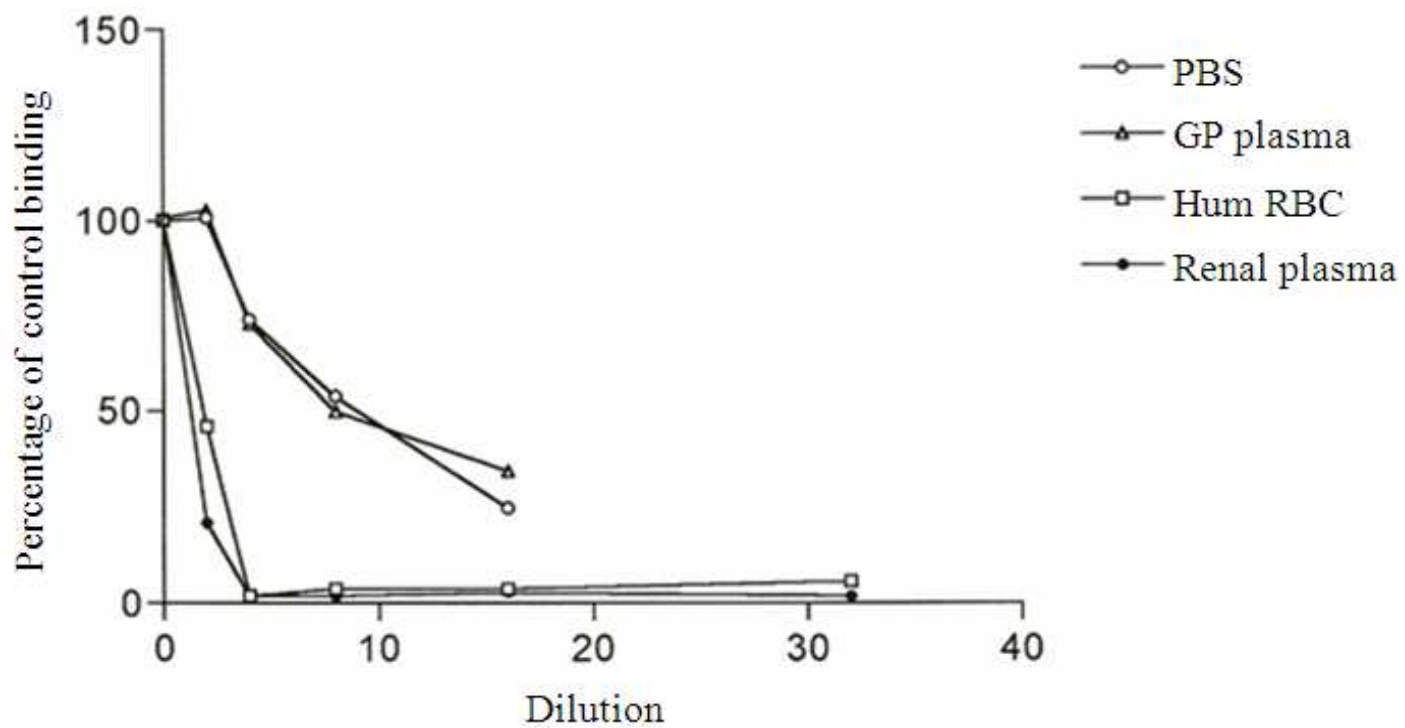

(A) 


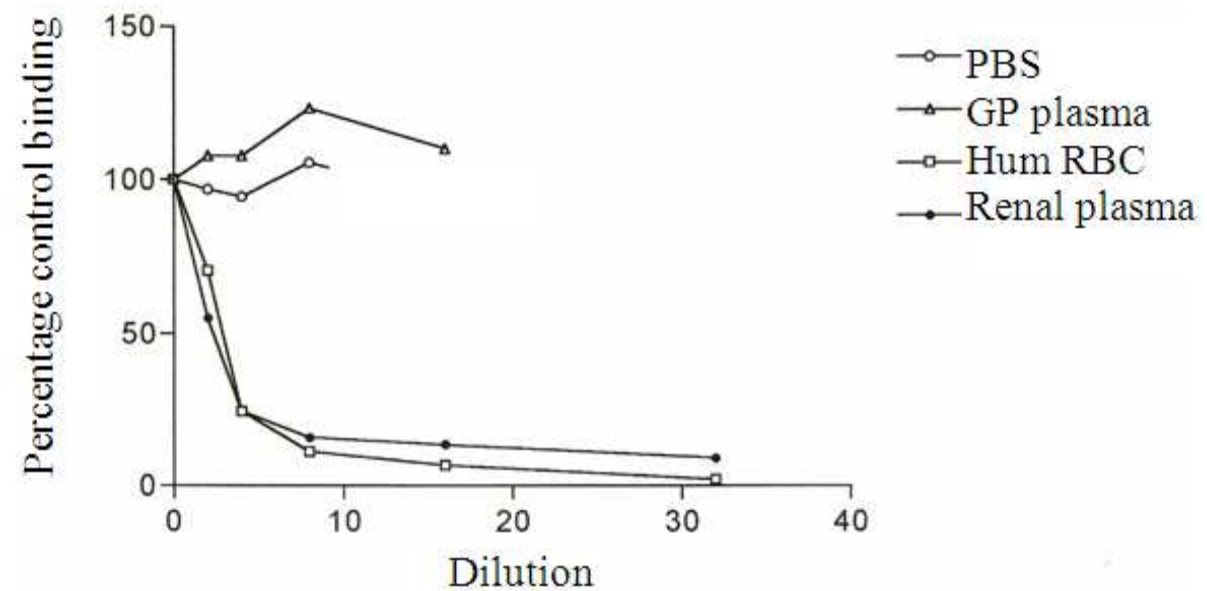

(B)

Fig. 3. (A) Competitive ELISA demonstrates specificity of binding of MEM-43 monoclonal antibody to CD59 by renal patient plasma. Erythrocyte membranes $(1 \mathrm{mg}$ protein $/ \mathrm{mL})$ as a known source of CD59 were coated onto microtiter plates. Monoclonal antibody MEM-43 was two-fold serially diluted into one of PBS buffer (10mM sodium phosphate pH 7.4, $150 \mathrm{mM} \mathrm{NaCl}$ ) (open circles), guinea pig plasma (open triangles), PBS containing $1 \mathrm{mg}$ protein/ml erythrocyte membranes (open squares) and pooled renal plasma (solid circles). Results are expressed as percentages of the binding of undiluted antibody. (B) Competitive ELISA demonstrates specificity of binding of YTH 53.1 monoclonal antibody to CD59 by renal patient plasma. Erythrocyte membranes $(1 \mathrm{mg}$ protein/ml $)$ as a known source of CD59 were coated onto microtiter plates. Monoclonal antibody YTH 53.1 to CD59 was two-fold serially diluted into PBS or into a competitor- containing solution or control solution consisting of guinea pig plasma (open triangles), PBS (open circles). PBS containing human erythrocytes (open squares) or renal plasma (solid circles). Reactivities of normal rat IgG (not shown) were $5 \%$ or less that of non-diluted YTH 53.1. Results are expressed as percentages of the binding of non-diluted antibody

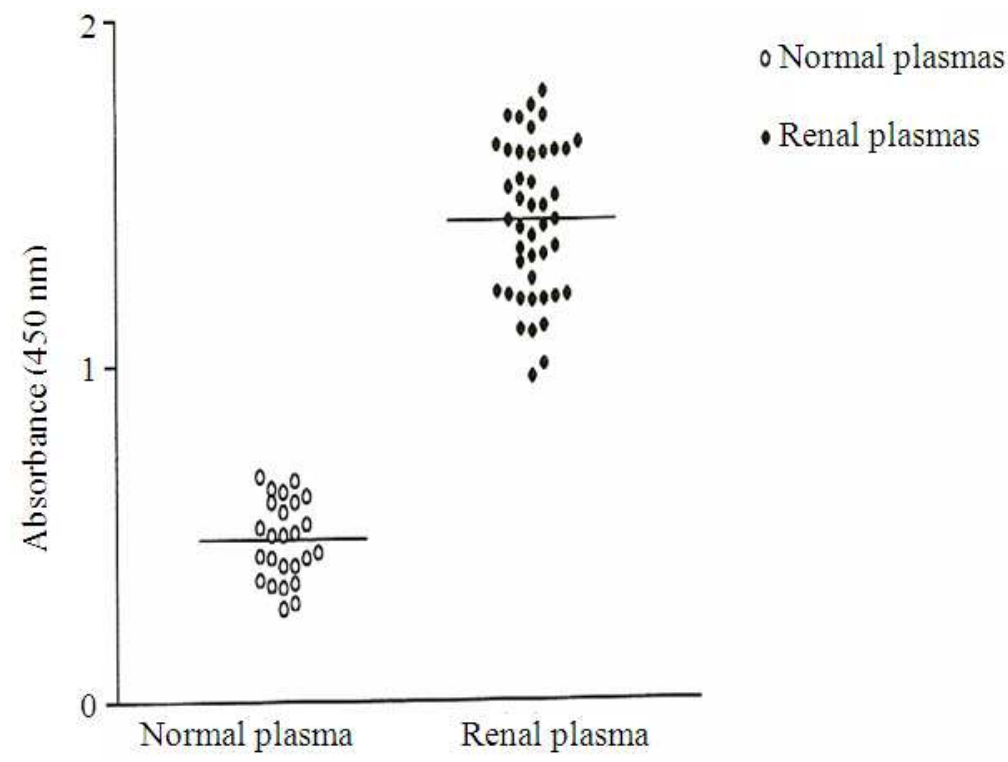

Fig. 4. Comparison of a larger number of samples shows consistently higher levels of CD59 content for different renal patient plasmas different normal plasmas. Plasmas from 25 different normal individuals (open circles) and 44 individuals with endstage renal disease (solid circles) were diluted twenty-fold and assessed for reactivity in sandwich ELISA with rabbit antiCD59 as capture antibody and monoclonal antibody MEM-43 to CD59 as detecting antibody. Points are means of duplicate absorbance values. The population means are indicated by horizontal lines 


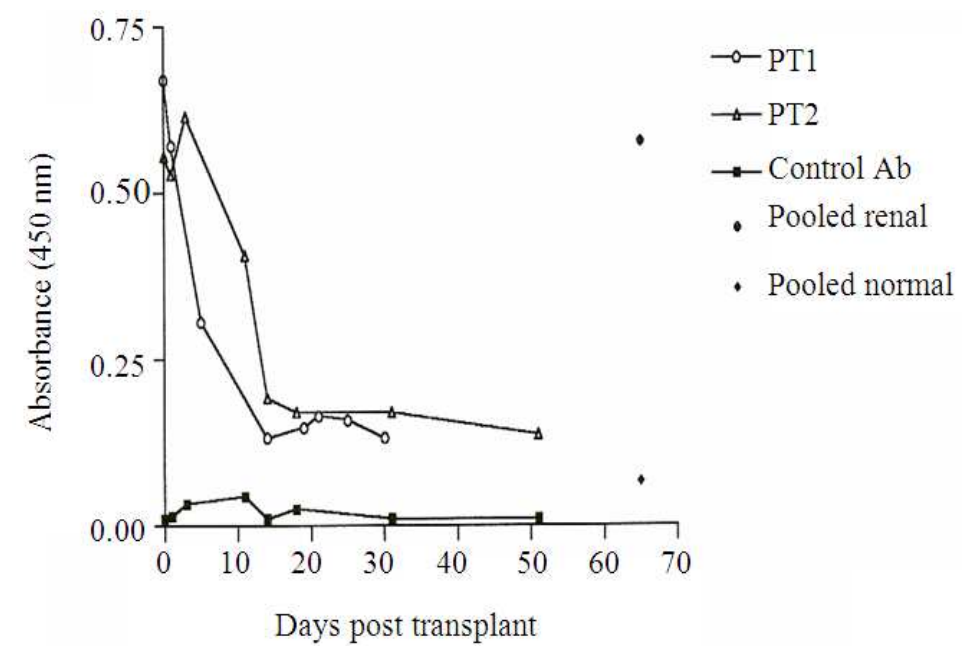

Fig. 5. CD59 levels decrease in plasmas from donors with end- stage renal disease after renal transplantation Plasmas from two individuals with end-stage renal disease, PT1 (open circles) and PT2 (triangles) were tested in CD59 sandwich ELISA before and at various days after renal transplantation. Values are means of duplicates for 20 -fold diluted plasma. Reactivity of control monoclonal antibody UPC-10 is shown (solid square). The solid diamond and solid circle) represent reactivities with MEM-43 of pooled normal and pooled renal patient plasmas respectively

Table 1. Plasma levels of soluble CD59 in heart, liver and lung transplantation candidates

\begin{tabular}{llr}
\hline Sample & $\begin{array}{l}\text { Absorbance } \\
(450 \mathrm{~nm})\end{array}$ & $\begin{array}{r}\text { (\%) of averaged } \\
\text { Normal Plasma }\end{array}$ \\
\hline PHS 1 & $0.546(+/-0.005)$ & 110 \\
PHS 2 & $0.450(+/-020)$ & 90 \\
Pooled & $1.187(+/-0.047)$ & 238 \\
Renal liver & $0.432(+/-0.20)$ & 87 \\
Average & & \\
$\mathrm{n}=4$ & \\
Heart & $0.359(+/-0.13)$ & \\
Average & & \\
$\mathrm{n}=5$ & & \\
Lung & $0.431(+/-0.001)$ & \\
\hline
\end{tabular}

Comparisons of leukemic and normal plasmas by the CD59 sandwich ELISA showed that in one case of Acute Leucocytic Leukemia (ALL), plasma reactivity with anti-CD59 was $195 \%$ that of normal plasma and plasmas from a second case of ALL and one from a donor with Chronic Myelogenous Leukemia (CML) were 117 and $99 \%$ that of normals respectively. Reactivity of plasma from one individual with lymphoma was $60 \%$ that of normal plasma. To confirm the soluble nature of plasma CD59-related antigen, untreated and ultracentrifuged plasma $(2 \mathrm{~h}, 160,000 \mathrm{~g})$ were compared for reactivity with anti-CD59. Absorbances of untreated plasma were $96 \%$ that of ultracentrifuged plasma. This finding suggested that plasma CD59-related antigen was not associated with membranous particles or cell fragments.

\subsection{Plasma CD59 in Patients Before and after Kidney Transplantation}

Sequential samples of plasmas from two individuals with end-stage renal disease were evaluated over several weeks to see if changes occurred in plasma reactivity with CD59 antibodies after renal transplantation (Fig. 5). The results showed a steady decline in plasma reactivity with CD59 antibodies after renal transplantation when compared with pre-transplant levels and pooled renal plasmas. Reactivities with anti-CD59 antibodies fell to a constant value in the plasma from the post transplant period but were still higher than that of pooled normal plasmas.

\section{DISCUSSION}

The present study reports the use of a sandwich ELISA to assay CD59 in plasmas from individuals with renal and disease. A major finding was that there were increased levels of CD59-related antigen in plasmas from individuals with end-stage renal disease. Another finding was that in two cases these higher levels decreased after kidney transplantation. Recognition of the CD59-related antigen by rabbit anti-CD59 and by two monoclonal antibodies to CD59 supports the possibility that CD59 and not a crossreacting molecule is being detected. 
Soluble CD59 has been reported to be present in several other body fluids but disease association was not found. The biological fluids that contained CD59 included urine, seminal plasma, saliva, tears, breast milk and amniotic fluid (Davies et al., 1989; Rooney and Morgan, 1993; Rooney et al., 1993). Amniotic fluid and seminal plasma are reported to contain soluble CD59 with an intact glycosylphosphatidyl inositol anchor, whilst CD59 in urine appears to lack a hydrophobic moiety (Davies et al., 1989; Rooney and Morgan, 1993; Rooney et al., 1993). Thus, CD59 has been found in various forms in different body fluids. Several studies now also support the existence of soluble forms of CD59 in blood. Soluble CD59 was reported to be present in retroplacental and umbilical cord and an immunoassay detected CD59 in sera (Bjorge et al., 1993; Landi et al., 2003). Consistent with the detection of reactivity of plasma and serum with anti-CD59 in the present study, CD59 was detected in the HDL fraction of plasma by immunoaffinity chromatography in low concentrations and purified CD59 was reported to associate with HDL (Vakeva et al., 1994). Our study provided some additional information that sera and plasma contained similar levels of CD59.

The cell or tissue origin of plasma CD59-related antigen is not known. Candidates include cells such as erythrocytes, leucocytes, platelets and endothelium which all express CD59 and are in contact with blood. Certain studies have identified conditions in which the expression of CD59 by cells is altered. For instance, myocardial infarction is accompanied by loss of CD59 from myocytes (Vakeva et al., 1992). In addition, soluble CD59 is increased in the acute sera from individuals suffering myocardial infarction (Vakeva et al., 2000). The cytokines, IL-1 and interferon gamma increase the expression of CD59 by thyroid epithelium (Tandon et al., 1992) of particular relevance to the present study, expression of CD59 was reported to be increased in the glomerular capillaries of kidneys from individuals with lupus nephritis (Tamai et al., 1991). In addition, the amount of soluble CD59 in urine was observed to be increased in membranous glomerulonephritis and urinary CD59 correlated with urinary SC5b-9 and IL-1 beta levels (Lehto et al., 1995). In the present study, increased reactivity with antibodies to CD59 was observed in plasmas from patients with renal disease relative to normals and individuals with failures of other organs and leukemias. This suggests further that the increased plasma CD59 was a phenomenon specific to renal disease among the conditions examined in this study. It is possible that damaged kidneys are the source or otherwise induce the increased plasma CD59. In two patients, the CD59-related antigen in plasma was decreased following renal transplantation and this suggested a link between renal malfunction and elevated plasma CD59-related antigen. Thus, although the mechanisms responsible for the increase in plasma CD59-related antigen are unknown, previous studies suggest tissue damage, cytokines and cellular activation as possible contributory factors and diseased kidneys as a possible source.

This study has provided additional evidence for the significance of CD59 in renal disease. It seems possible that understanding how soluble forms of CD59 is produced and how its levels are regulated will provide more insight into renal disease processes.

\section{CONCLUSION}

This study found that soluble CD59 levels are increased in plasmas from individuals with end stage renal disease but not in other conditions such as leukemia, lung and liver disease. It was also found that soluble CD59 decreased from elevated levels after kidney transplantation in two cases.

\section{ACKNOWLEDGEMENT}

Dr CJ Parker is thanked for the kind gift of rabbit anti-CD59. Much of the work was done at Methodist Hospital of Indiana.

\section{REFERENCES}

Bao, L., M. Haas, J. Pippin, Y. Wang and T. Miwa et al., 2009. Focal and segmental glomerulosclerosis induced in mice lacking decay-accelerating factor in T cells. J. Clin. Investigat., 119: 1264-74. DOI: $10.1172 / \mathrm{JCI} 36000$

Berger, S.P. and M.R. Daha, 2007. Complement in glomerular injury. Seminars Immunopathol., 29: 375-382. DOI: 10.1007/s00281-007-0090-3

Bjorge, L., T.S. Jensen, C.A. Vedeler, E. Ulvestad and E.K. Kristofferson et al., 1993. Soluble CD59 in pregnancy and infancy. Immunol. Lett., 36: 233234. DOI: $10.1016 / 0165-2478(93) 90058-A$

Davies, A., D.L. Simmons, G. Hale, R.A. Harrison and H. Tighe et al., 1989. CD59, an LY-6-like protein expressed in human lymphoid cells, regulates the action of the complement membrane attack complex on homologous cells. J. Exp. Med., 170: 637-654. PMID: 2475570

He, C., M. Imai, H. Song, R.J. Quigg and S. Tomlinson, 2005. Complement inhibitors targeted to the proximal tubule prevent injury in experimental nephrotic syndrome and demonstrate a key role for C5b-9. J. Immunol., 1: 5750-5757. PMID: 15843577 
Heeringa, S.F. and C.D. Cohen, 2012. Kidney diseases caused by complement dysregulation: Acquired, inherited and still more to come. Clin. Developm. Immunol. DOI: 10.1155/2012/695131

Landi, A.P., A.B. Wilson, A. Davies, P.J. Lachmann and V.P. Ferriani et al., 2003. Determination of CD59 protein in normal human serum by enzyme immunoassay, using octyl-glucoside detergent to release glycosyl-phosphatidylinositol-CD59 from lipid complex. Immunol. Lett., 90: 209-213. DOI: 10.1016/j.imlet.2003.07.001

Lehto, T., E. Honkanen, A.M. Teppo and S. Meri, 1995. Urinary excretion of protectin (CD59), complement SC5b-9 and cytokines in membranous glomerulonephritis. Kidney Int., 47: 1043-1051. DOI: $10.1038 / \mathrm{ki} .1995 .197$

Miwa, T., S. Sato, D. Gullipalli, M. Nangaku and W.C. Song, 2013. Blocking properdin, the alternative pathway and anaphylatoxin receptors ameliorates renal ischemia-reperfusion injury in decayaccelerating factor and CD59 double-knockout mice. J. Immunol., 190: 3552-3559. DOI: 10.4049/jimmunol.1202275. Epub 2013 Feb 20

Nishi, S., N. Imai, Y. Ito, M. Ueno and S. Fukase et al., 2004. Pathological study on the relationship between C4d, CD59 and C5b-9 in acute renal allograft rejection. Clin. Transplantation, 18: 18-23. DOI: 10.1111/j.1399-0012.2004.00242

Nishi, S., N. Immai, Y. Ito, M. Ueno and S. Fukase et al., 2013. Shiga toxin 2 reduces complement inhibitor CD59 expression on human renal tubular epithelial and glomerular endothelial cells. Infect. Immunity. DOI: 10.1128/IAI.01079-12

Qi, K., X.G. Zhang, S.W. Liu, Z. Yin and X.M. Chen et al., 2011. Reversible acute kidney injury caused by paroxysmal nocturnal hemoglobinuria. Am. J. Med. Sci., $\quad 341$ : $\quad 68-70 . \quad$ DOI: 10.1097/MAJ.0b013e3181f515b9

Rooney, I.A. and B.P. Morgan, 1993. Characterization of the membrane attack complex inhibitory protein CD59 antigen on human amniotic cells and in amniotic fluid. Immunology, 76: 541-547. PMID: 1383132

Rooney, I.A., J.P. Atkinson, E.S. Krul, G. Schonfeld and K.M. Polakoski et al., 1993. Physiologic relevance of the membrane attack complex inhibitory protein CD59 in human seminal plasma: CD59 is present on extracellular organelles (prostasomes), binds cell membranes and inhibits complement-mediated lysis. J. Exp. Med., 177: 1409-1420. PMID: 7683035
Tamai, H., S. Matsuo, A. Fukatsu, K. Nishikawa and N. Sakamoto et al., 1991. Localization of $20-\mathrm{kD}$ homologous restriction factor (HRF20) in diseased human glomeruli. An immunofluorescence study. Clin. Exp. Immunol., 84: 256-262. PMID: 1709070

Tandon, R., J.E. Shipley, S. Taylor, and J.F. Greden, 1992. Sleep abnormalities in schizophrenia: Cholinergic contribution. Clin. Neuropharmacol., 15: 294A-295A. PMID: 1498847

Tembhare, P., M. Ramani, K. Syed and A.D. Gupta, 2010. Flow cytometric analysis of erythrocytes in paraoxysmal nocturnal hemoglobinuria reveals superiority of CD59 as a diagnostic marker compared to CD55. Indian J. Pathol. Microbiol., 53: 699-703. DOI: 10.4103/0377-4929.72042

Vakeva, A., M. Jauhiainen, C. Ehnholm, T. Lehto and S. Meri, 1994. High-density lipoproteins can act as carriers of glycophosphoinositol lipid-anchored CD59 in human plasma.. Immunology, 82: 28-33. PMID: 7519171

Vakeva, A., P. Laurila and S. Meri, 1992. Loss of expression of protectin (CD59) is associated with complement membrane attack complex deposition in myocardial infarction. Lab. Invest., 67: 608-616. PMID: 1279272

Vakeva, A., T. Lehto, A. Takala and S. Meri, 2000. Detection of a soluble form of the complement membrane attack complex inhibitor CD59 in plasma after acute myocardial infarction. Scand. J. Immunol., 52: 411-414. DOI: 10.1046/j.13653083.2000.00783.x

Vanderpuye, O.A., C.A. Labarrere and J.A. McIntyre, 1993. Expression of CD59, a human complement system regulatory protein in extraembryonic membranes. Int. Archiv. Allergy Immunol., 101: 376-384. DOI: $10.1159 / 000236480$

Xu, L., W. Wu, Z. Zhao, H. Shao and W. Liu et al., 2006. Cooperation between human DAF and CD59 in protecting cells from human complementmediated lysis. J. Biochem. Mol. Biol., 39: 743-8. PMID: 17129411

Yang, X., J. Deng, Z. Jiang, D.J. Liao and H.C. Jiang, 2011. Protective effects of different combinations of human MCP, DAF and CD59 on complementdependent cytolysis in NIH $3 \mathrm{~T} 3$ cells. Exp. Clin. Transplant., 10: 49-54. DOI: 10.6002/ect.2011.0097

Zipfel, P.F. and C. Skerka, 2009. Complement regulators and inhibitory proteins. Nature Rev. Immunol., 9: 729-740. DOI: $10.1038 /$ nri2620

Zhu, X., M. Gao, S. Rens, Q. Wang and C. Lin, 2008. Activity after site-directed mutagenesis of CD59 on complement-mediated cytolysis. Cellul. Molec. Immunol., 5: 141-146. PMID: 18445344 